\title{
Síndrome de Burnout em profissionais de enfermagem durante a pandemia de Covid- 19 em um município no Sudoeste do Pará
}

\author{
Burnout syndrome in nursing professionals during the Covid-19 pandemic in a municipality in the \\ Southwest of Pará \\ Síndrome de Burnout en profesionales de enfermería durante la pandemia de Covid-19 en un \\ municipio del suroeste de Pará
}

Recebido: 10/08/2021 | Revisado: 18/08/2021 | Aceito: 22/11/2021 | Publicado: 29/11/2021

\author{
Valdino Santana Campos Junior \\ ORCID: https://orcid.org/0000-0003-0118-7834 \\ Universidade Luterana do Brasil, Brasil \\ E-mail: juniorcampos_28@hotmail.com \\ Ana Maria Pujol Vieira dos Santos \\ ORCID: https://orcid.org/0000-0001-9025-5215 \\ Universidade Luterana do Brasil, Brasil \\ E-mail: anapujol@ulbra.br \\ André Guirland Vieira \\ ORCID: https://orcid.org/0000-0001-9098-6253 \\ Universidade Luterana do Brasil, Brasil \\ E-mail: andre.vieira@ulbra.br
}

\begin{abstract}
Resumo
A síndrome de Burnout (SB) está presente na vida de vários profissionais da saúde, pois estão expostos a diversas situações de estresse no ambiente de trabalho. O objetivo da pesquisa foi descrever a SB em enfermeiros e técnicos de enfermagem atuantes em Unidades Básicas de Saúde e Hospital em um município no Sudoeste do Pará. A amostra foi composta por 109 profissionais, dos quais 49 eram enfermeiros e 60 técnicos de enfermagem. Foram utilizados dois instrumentos de coleta de dados: questionário sociodemográfico e de saúde e o questionário Maslach Burnout Inventory (MBI). Para verificar a associação entre a classificação da síndrome de Burnout com dados sociodemográficos foi utilizado o teste Qui Quadrado ou exato de Fischer de acordo com as suposições dos testes. A SB esteve presente em $12,8 \%$ participantes, destes $64,3 \%$ são enfermeiros. Com relação as dimensões do MBI, 59,9\% apresentaram baixa realização profissional, $42,2 \%$ alta exaustão emocional e 58,7\% moderada despersonalização. A dimensão exaustão emocional foi a que apresentou maior percentual no nível alto, quando comparada com despersonalização $(27,5 \%)$ e a realização profissional $(2,8 \%)$. Não foram identificadas diferenças estatísticas na associação das dimensões com os dados sociodemográficos. A exaustão profissional foi associada ao realizar tratamento para depressão $(\mathrm{p}=0,05)$, praticar atividade física $(\mathrm{p}=0,01)$, sentimento em relação ao trabalho $(\mathrm{p}=0,04)$ e ter sofrido algum tipo de violência no trabalho $(\mathrm{p}=0,03)$. A despersonalização foi associada a religião $(\mathrm{p}=0,01)$, sentimento em relação ao trabalho $(\mathrm{p}=0,02)$, ter sofrido algum tipo de violência no trabalho $(\mathrm{p}=0,01)$ e acreditar ser afetado pela SB $(\mathrm{p}=0,03)$, enquanto a realização pessoal foi associada religião $(\mathrm{p}=0,03)$. O desenvolvimento de ações voltadas para a promoção da saúde e bem-estar desses profissionais é importante considerando o momento de pandemia de Covid-19.
\end{abstract}

Palavras-chave: Serviços de saúde; Esgotamento psicológico; Enfermagem; Covid-19.

\section{Abstract}

Burnout syndrome (BS) is present in the lives of many health professionals, as they are exposed to different situations of stress in the work environment. The objective of the research was to describe the BS in nurses and nursing technicians working in Basic Health Units and Hospitals in a city in the Southwest of Pará. The sample consisted of 109 professionals, of which 49 were nurses and 60 nursing technicians. Two data collection instruments were used: sociodemographic and health questionnaire and the Maslach Burnout Inventory (MBI) questionnaire. To verify the association between the classification of Burnout syndrome and sociodemographic data, the Chi-Square or Fisher's exact test was used, according to the test assumptions. SB was present in $12.8 \%$ of the participants, of which $64.3 \%$ are nurses. Regarding the dimensions of the MBI, $59.9 \%$ had low professional achievement, $42.2 \%$ had high emotional exhaustion and $58.7 \%$ had moderate depersonalization. The emotional exhaustion dimension was the one with the highest percentage at the high level, when compared to depersonalization (27.5\%) and professional fulfillment (2.8\%). No statistical differences were identified in the association of dimensions with sociodemographic data. Professional exhaustion was associated with undergoing treatment for depression $(\mathrm{p}=0.05)$, practicing physical activity $(\mathrm{p}=0.01)$, feeling about work $(\mathrm{p}=0.04)$ and having suffered some type of violence at work $(\mathrm{p}=0.03)$. Depersonalization was associated with religion 
$(\mathrm{p}=0.01)$, feeling towards work $(\mathrm{p}=0.02)$, having suffered some type of violence at work $(\mathrm{p}=0.01)$ and believing to be affected by BS $(\mathrm{p}=0.03)$, while personal fulfillment was associated with religion $(\mathrm{p}=0.03)$. The development of actions aimed at promoting the health and well-being of these professionals is important considering the Covid-19 pandemic moment.

Keywords: Health services; Psychological exhaustion; Nursing; Covid-19.

\section{Resumen}

El síndrome de Burnout (SB) está presente en la vida de muchos profesionales de la salud, ya que están expuestos a diferentes situaciones de estrés en el entorno laboral. El objetivo de la investigación fue describir el BS en enfermeros y técnicos de enfermería que laboran en Unidades Básicas de Salud y Hospitales de una ciudad del Suroeste de Pará, la muestra estuvo conformada por 109 profesionales, de los cuales 49 eran enfermeros y 60 técnicos de enfermería. Se utilizaron dos instrumentos de recolección de datos: cuestionario sociodemográfico y de salud y cuestionario Maslach Burnout Inventory (MBI). Para verificar la asociación entre la clasificación del síndrome de Burnout y los datos sociodemográficos se utilizó la prueba de Chi-Cuadrado o la prueba exacta de Fisher, según los supuestos de la prueba. SB estuvo presente en el 12,8\% de los participantes, de los cuales el 64,3\% son enfermeros. En cuanto a las dimensiones del MBI, el 59,9\% tenía bajo rendimiento profesional, el 42,2\% tenía alto agotamiento emocional y el 58,7\% tenía una despersonalización moderada. La dimensión de agotamiento emocional fue la de mayor porcentaje en el nivel alto, en comparación con la despersonalización $(27,5 \%)$ y la realización profesional $(2,8 \%)$. No se identificaron diferencias estadísticas en la asociación de dimensiones con datos sociodemográficos. El agotamiento profesional se asoció a recibir tratamiento por depresión $(\mathrm{p}=0,05)$, practicar actividad física $(\mathrm{p}=0,01)$, sentir el trabajo $(\mathrm{p}=0,04)$ y haber sufrido algún tipo de violencia en el trabajo $(\mathrm{p}=0,03)$. La despersonalización se asoció a la religión $(\mathrm{p}=0,01)$, sentimiento hacia el trabajo $(p=0,02)$, haber sufrido algún tipo de violencia en el trabajo $(p=0,01)$ y creerse afectado por $B S(p=0,03)$, mientras que la realización personal fue asociado a la religión $(\mathrm{p}=0,03)$. El desarrollo de acciones dirigidas a promover la salud y el bienestar de estos profesionales es importante considerando el momento de la pandemia Covid-19.

Palabras clave: Servicios de salud; Agotamiento psicológico; Enfermería; Covid-19.

\section{Introdução}

A rotina cansativa dos profissionais da saúde contribui diretamente com o grau de estresse causando sérias consequências. A síndrome de Burnout (SB) é reconhecida como um risco ocupacional para profissões que envolvem cuidados com saúde, educação e serviços humanos (Trigo, Teng, \& Hallak, 2007). É decorrente do estresse ocupacional crônico, afetando aspectos da vida individual, profissional e familiar. Este estresse pode acarretar consequências ao trabalhador como a perda da capacidade de se adaptar às demandas existentes no contexto laboral (Tavares, Souza, Silva, \& Kestenberg, 2014).

Segundo a Associação Nacional de Medicina do Trabalhador - ANAMT (2018), 30\% dos mais de 100 milhões de trabalhadores brasileiros sofrem com SB. Essa síndrome tem relação direta com exaustão emocional, dores de cabeça e musculares que ocorrem com frequência e geram reclamações dos profissionais, e os trabalhos excessivos são algumas das causas que geram os sintomas associados. O constante estresse do cotidiano dos profissionais de enfermagem é considerado uma das principais incidências dentro do atual contexto, pois eles se enquadram em uma rotina de trabalho cansativa. Enfermeiros e técnicos sofrem influência contínua de diversos estressores do ambiente de trabalho, como carga horária fatigante, quadro de funcionários reduzido, baixa remuneração, ausência de equipamentos de proteção e a complexidade dos procedimentos, fatores que são determinantes para o desenvolvimento da SB (Vasconcelos, De Martino, \& França, 2018, Vilaço, Gonçalves, Silva, \& Vetorazo, 2021). Soma-se que os profissionais de enfermagem enfrentam diariamente situações diferentes e complexas no ambiente de trabalho. Essas situações envolvem sua capacidade de cuidar bem, como estar atento ao meio em que estão inseridos. Quando estão em suas atividades laborais, ficam expostos ao estresse ocupacional, que possui diversas origens e de alguma forma afeta diretamente o profissional (Vasconcelos \& De Martino, 2017).

Acrescenta-se a este cenário o ano de 2020, que foi desafiador para a ciência, para economia e para a sociedade, com o surgimento do vírus Sars-Cov-2. Esse novo vírus teve seus primeiros casos descobertos na China, em dezembro de 2019, através de sintomas como tosse, febre, perda de olfato e paladar, sendo capaz de causar infecções respiratórias graves. Com o avanço de casos de Covid-19 no mundo, a Organização Mundial da Saúde declarou o estado de pandemia em 11 de março de 2020 e o fato foi noticiado em todos os meios de comunicação e passou a ser notícia e motivo de preocupação para toda a população. O novo 
coronavírus foi capaz de atingir, em menos de três meses, cento e quatorze países, dentre eles o Brasil. A rápida disseminação do vírus adveio do seu altíssimo poder de contágio (Brasil, 2020). Através do avanço da pandemia e das medidas de restrição e isolamento social impostas pela Covid-19, houve um impacto direto em todos os segmentos da sociedade, especialmente nos profissionais de enfermagem (Soares et al., 2021).

No entanto, com a chegada da pandemia frequentemente a discussão na mídia concentrou-se no número de ventiladores e leitos hospitalares, muitas vezes faltando discutir sobre como estão os números de profissionais da saúde disponíveis e qualificados. Ainda, deveria haver uma preocupação não apenas com o número de profissionais qualificados, mas também se estes estão saudáveis, mentalmente e fisicamente, para fazer o atendimento dos pacientes (Ross, 2020). É essencial reconhecer como o estresse relacionado ao local de trabalho pode afetar o bem-estar mental e emocional. Esses estressores podem impactar ainda mais os contextos de recursos limitados, resultando em uma grave escassez de profissionais de saúde (Sultana, Sharma, Hossain, Bhattacharya, \& Purohit, 2020).

No Brasil, durante a pandemia, as condições laborais adversas dos enfermeiros, em especial no que se refere a falta de profissionais, sobrecarga de atividades, prolongadas horas de trabalho, baixa remuneração e falta/inadequação de equipamentos de proteção individual, risco da própria contaminação e isolamento podem levar a exaustão (Backes et al., 2021, Horta et al., 2021, Souza et al., 2021).

Dentro da realidade desses profissionais é importante o levantamento de dados sobre a SB e seus aspectos relacionados. Nesse sentido, o objetivo deste trabalho foi identificar a SB em enfermeiros e técnicos de enfermagem atuantes em Unidades Básicas de Saúde e Hospital em um município no Sudoeste do Pará.

\section{Metodologia}

Este trabalho é caracterizado como um estudo transversal, analítico com abordagem quantitativa, tendo sido realizado em um município da região Norte do Brasil, pertencente à Mesorregião do Sudoeste Paraense, em abril de 2021.

Participaram da pesquisa enfermeiros e técnicos de enfermagem que atuam há mais de seis meses nas 15 Unidades Básicas de Saúde (UBS) e no Hospital Municipal. Foram excluídos profissionais em férias ou de licença maternidade. A seleção dos participantes ocorreu por conveniência sendo composta por 109 profissionais, 49 enfermeiros e 60 técnicos de enfermagem, de um total de 53 enfermeiros e 99 técnicos.

Para a realização do estudo, após o aceite da Secretaria de Saúde do município, foi solicitada a relação dos trabalhadores do Hospital Municipal e das 15 UBS. Os participantes foram contatados individualmente no seu local de trabalho pelo pesquisador e convidados a participar do estudo.

Foram utilizados dois questionários. Para caracterização da população, foi utilizado um questionário com 32 perguntas sobre dados pessoais, socioeconômicos, enfermidades, hábitos saudáveis, saúde mental e ambiente de trabalho. Para identificar a SB, foi utilizado o Maslach Burnout Inventory (MBI) A versão atual é composta por 22 perguntas relacionadas aos profissionais no ambiente de trabalho e seu estado emocional (Malasch \& Jackson, 1986) e foi traduzido e validado para a língua portuguesa por Lautert (1995). As respostas são em escala Likert, com as seguintes opções: 1 - Nunca; 2 - Algumas vezes no ano; 3 Algumas vezes no mês; 4 - Algumas vezes na semana; 5 - Diariamente. O preenchimento deste questionário leva em média de 10 a 15 minutos. É composto por três dimensões: Exaustão Emocional, Despersonalização e a Realização Profissional. A exaustão emocional é composta por nove questões $(1,2,3,6,8,13,14,16$, e 20), que traduzem sentimentos de se estar emocionalmente exausto e esgotado com o trabalho. A despersonalização é formada por cinco itens (5, 10, 11, 15 e 22), relacionados aos sentimentos negativos em relação ao próximo e às atitudes de ironia e cinismo com o outro e a realização profissional é constituída por oito questões $(4,7,9,12,17,18,19$ e 21), que descrevem sentimentos ao nível da capacidade e sucessos alcançados no trabalho com pessoas. Foram utilizados os seguintes pontos de corte: exaustão emocional (baixo: menor/igual a 16; moderado: 17 
a 26; alto: maior/igual a 27); despersonalização (baixo: menor/igual a 6; moderado: 7 a 12; alto: maior/igual a 13); realização profissional (baixo: menor/igual a 31; moderado: 32 a 38; alto: maior/igual a 39). Constata-se a SB pela combinação de escores altos em exaustão emocional e despersonalização e escores baixos na realização profissional (Lautert, 1995). A consistência interna foi verificada por meio do coeficiente alfa de Cronbach, cujos valores foram de 0,65 para despersonalização, 0,70 para exaustão emocional, 0,73 para realização profissional e 0,81 fator global. Todos os itens foram considerados pertinente para mensurar os itens da escala de Burnout já que, no geral, os valores de alfa de Cronbach ficaram acima de 0,70, que é considerado o mínimo aceitável.

Os dados foram digitados no software Epidata e posteriormente exportados para o Microsoft Excel 2010. A análise de dados foi realizada considerando os objetivos propostos para o estudo. Os dados receberam tratamento estatístico, sendo que os resultados das variáveis nominais foram expressos através de análises de frequência relativa e absoluta. Para verificar a associação entre a classificação da SB com dados sociodemográficos foram utilizados o teste Qui Quadrado ou exato de Fischer, de acordo com as suposições dos testes. Quando essas não foram atendidas, em relação às frequências esperadas, foi utilizado o teste exato de Fischer, conforme o algoritmo de Mehta e Patel (1980), que possibilita a aplicabilidade do teste exato de Fisher para tabelas de dimensões maiores que $2 \times 2$, onde as suposições do teste $\chi 2$ não forem satisfeitas. Foi considerado um $p \leq 0,05$ como significância estatística.

A pesquisa foi aprovada pelo Comitê de Ética em Pesquisa da Universidade Luterana do Brasil em março de 2021 (CAAE 44542821.4.0000.5349), sendo realizada conforme as Diretrizes e Normas Reguladoras de Pesquisa envolvendo Seres Humanos, estabelecido pela resolução 466/2012 do Conselho Nacional de Saúde.

\section{Resultados}

Os participantes da pesquisa eram na maioria do sexo feminino (82,6\%). A idade apresentou uma variação de 20 a 62 anos, com média de 36,92 anos $( \pm 8,70)$, a cor/etnia predominante foi a parda $(85,3 \%)$ e o estado civil foi o solteiro (44\%).

Para verificar a SB foi utilizada a pontuação MBI e no presente estudo quatorze profissionais $(12,8 \%)$ atendiam ao critério de escores altos em exaustão emocional e despersonalização e escores baixos na realização profissional. Destes, nove eram enfermeiros e cinco técnicos. Ao associar os dados sociodemográficos, de percepção de ansiedade, depressão e hábitos de saúde (Tabela 1) e dados do ambiente laboral (Tabela 2) com a presença de SB, não foram encontradas diferenças estatísticas ( $\mathrm{p} \geq 0,05$ ). Entretanto, pode-se observar a prevalência da SB nos enfermeiros, sexo feminino, casados/união estável, que não praticavam atividade física, não fumavam e não consumiam bebidas alcoólicas.

Tabela 1. Associação entre a ocorrência da síndrome de Burnout com dados sociodemográficos, percepção de ansiedade, depressão e hábitos de saúde de profissionais de enfermagem de uma cidade do oeste Paraense (2021).

\begin{tabular}{lccc}
\hline Variáveis & Não & Sim & Valor de p \\
\hline Idade: (a) $^{\mathbf{1}}$ & $36,94 \pm 8,81$ & $36,78 \pm 8,22$ & 0,09 \\
Profissão: $^{2}$ & $55(91,7 \%)$ & $5(8,3 \%)$ & 0,06 \\
Técnico de Enfermagem $^{\text {Enfermeiro }}$ & $40(81,6 \%)$ & $9(18,4 \%)$ & \\
Sexo: ${ }^{2}$ & & & 0,45 \\
Feminino & $77(85,6 \%)$ & $13(14,4 \%)$ & \\
Masculino & $18(94,7 \%)$ & $1(5,3 \%)$ & \\
Cor/Etnia: ${ }^{2}$ & & & 0,79 \\
Branca & $7(77,8 \%)$ & $2(22,2 \%)$ & \\
Parda & $82(88,2 \%)$ & $11(11,8 \%)$ & \\
Negra & $5(83,3 \%)$ & $1(16,7 \%)$ & \\
& &
\end{tabular}


Research, Society and Development, v. 10, n. 15, e458101519274. 2021

(CC BY 4.0) | ISSN 2525-3409 | DOI: http://dx.doi.org/10.33448/rsd-v10i15.19274

Quilombola

Estado Civil: ${ }^{2}$

Solteira $(o)$

Casada(o)

Separada(o)

Divorciada(o)

Viúva(o)

União Estável

Renda mensal individual: ${ }^{2}$

Até $1 \mathrm{SM}(\mathrm{R} \$ 1.100)$

Até $2 \mathrm{SM}(\mathrm{R} \$ 2.200)$

Até $3 \mathrm{SM}(\mathrm{R} \$ 3.300)$

De 3 à 5 SM (R\$3.30 à 5.500)

Acima de 5 SM $(\mathrm{R} \$ 5,500)$

Escolaridade: ${ }^{2}$

Médio Completo

Superior Incompleto

Superior Completo

Especialização

Mestrado

Você já realizou tratamento para ansiedade: ${ }^{2}$

Não

Sim

Você já realizou tratamento para depressão: ${ }^{2}$

Não

Sim

Consome bebida alcoólica: ${ }^{2}$

Não

Sim

Fuma: ${ }^{2}$

Não

Sim

Pratica atividade física: ${ }^{2}$

Não

Sim
$1(100 \%) \quad 0(0 \%)$

$44(91,7 \%) \quad 4(8,3 \%)$

$25(80,6 \%) \quad 6(19,4 \%)$

$3(100 \%) \quad 0(0 \%)$

$3(75 \%) \quad 1(25 \%)$

$0(0 \%) \quad 0(0 \%)$

$20(87 \%) \quad 3(13 \%)$

0,52

$\begin{array}{ll}23(85,2 \%) & 4(14,8 \%) \\ 35(94,6 \%) & 2(5,4 \%) \\ 16(84,2 \%) & 3(15,8 \%) \\ 15(78,9 \%) & 4(21,1 \%) \\ 6(85,7 \%) & 1(14,3 \%)\end{array}$

0,83

$25(86,2 \%) \quad 4(13,8 \%)$

$14(93,3 \%) \quad 1(6,7 \%)$

$18(90 \%) \quad 2(10 \%)$

$36(83,7 \%) \quad 7(16,3 \%)$

$2(100 \%) \quad 0(0 \%)$

0,57

$85(86,7 \%) \quad 13(13,3 \%)$

$10(90,9 \%) \quad 1(9,1 \%)$

$91(86,7 \%) \quad 14(13,3 \%)$

$4(100 \%) \quad 0(0 \%)$

$46(85,2 \%) \quad 8(14,8 \%)$

$49(89,1 \%) \quad 6(10,9 \%)$

$93(86,9 \%) \quad 14(13,1 \%)$

$2(100 \%) \quad 0(0 \%)$

$51(85 \%) \quad 9(15 \%)$

$44(89,8) \quad 5(10,2 \%)$

(a) Resultados expressos através de média \pm desvio padrão. SM-Salário-Mínimo.

Demais resultados expressos através de análises de frequência

$\left({ }^{1}\right)$ Teste $t$ para amostras independentes $\left({ }^{2}\right)$ Teste exato de Fischer

Fonte: Dados da pesquisa. 
Tabela 2 - Associação entre a ocorrência de Burnout com dados do ambiente laboral de profissionais de enfermagem de uma cidade do oeste Paraense (2021).

\begin{tabular}{|c|c|c|c|}
\hline Variáveis & $\begin{array}{c}\text { Não } \\
\mathrm{n}=95\end{array}$ & $\begin{array}{c}\operatorname{Sim} \\
\mathrm{n}=14\end{array}$ & Valor de $\mathrm{p}$ \\
\hline $\begin{array}{l}\text { Há quanto tempo você trabalha como } \\
\text { enfermeiro ou técnico de enfermagem: }\end{array}$ & & & 0,52 \\
\hline Entre 6 meses e 1 ano & $7(77,8 \%)$ & $2(22,2 \%)$ & \\
\hline Entre 1 e 2 anos & $14(87,5 \%)$ & $2(12,5 \%)$ & \\
\hline Entre 2 e- 4 anos & $14(82,4 \%)$ & $3(17,6 \%)$ & \\
\hline Entre 4 e 6 anos & $19(100 \%)$ & $0(0 \%)$ & \\
\hline Entre 6 e 8 anos & $10(100 \%)$ & $0(0 \%)$ & \\
\hline Mais de 8 anos & $31(81,6 \%)$ & $7(18,4 \%)$ & \\
\hline Qual ambiente você trabalha: & & & 0,53 \\
\hline Emergência & $17(81 \%)$ & $4(19 \%)$ & \\
\hline Internação & $15(83,3 \%)$ & $3(16,7 \%)$ & \\
\hline Obstetrícia & $3(100 \%)$ & $0(0 \%)$ & \\
\hline Centro cirúrgico & $13(100 \%)$ & $0(0 \%)$ & \\
\hline Outro & $47(87 \%)$ & $7(13 \%)$ & \\
\hline $\begin{array}{l}\text { Qual a seu sentimento em relação ao } \\
\text { seu trabalho: }\end{array}$ & & & 0,67 \\
\hline Muito satisfeito & $13(92,9 \%)$ & $1(7,1 \%)$ & \\
\hline Satisfeito & $44(88 \%)$ & $6(12 \%)$ & \\
\hline Pouco Satisfeito & $28(84,8 \%)$ & $5(15,2 \%)$ & \\
\hline Insatisfeito & $8(88,9 \%)$ & $1(11,1 \%)$ & \\
\hline Muito insatisfeito & $2(66,7 \%)$ & $1(33,3 \%)$ & \\
\hline Ha quanto tempo não tira Férias: & & & 0,87 \\
\hline Menos de um ano & $17(89,5 \%)$ & $2(10,5 \%)$ & \\
\hline Um ano & $5(83,3 \%)$ & $1(16,7 \%)$ & \\
\hline Mais de um ano. & $73(86,9 \%)$ & $11(13,1 \%)$ & \\
\hline $\begin{array}{l}\text { Em 2020, você esteve ausente por } 2 \text { dias } \\
\text { ou mais: }\end{array}$ & & & 0,33 \\
\hline Não & $30(90,9 \%)$ & $3(9,1 \%)$ & \\
\hline Sim & $65(85,5 \%)$ & $11(14,5 \%)$ & \\
\hline $\begin{array}{l}\text { Nos últimos } 12 \text { meses, você sofreu } \\
\text { algum tipo de violência no seu } \\
\text { trabalho: }\end{array}$ & & & 0,58 \\
\hline Não & $46(85,2 \%)$ & $8(14,8 \%)$ & \\
\hline $\operatorname{Sim}$ & $49(89,1 \%)$ & $6(10,9 \%)$ & \\
\hline $\begin{array}{l}\text { Você acha que é afetado por esta } \\
\text { síndrome: }\end{array}$ & & & 0,33 \\
\hline Não & $76(88,4)$ & $10(11,6 \%)$ & \\
\hline Sim & $19(82,6 \%)$ & $4(17,4 \%)$ & \\
\hline
\end{tabular}

Resultados expressos através de análises de frequência. Teste exato de Fischer Fonte: Dados da pesquisa.

As dimensões do instrumento MBI são apresentadas na Figura 1. Houve predominância de baixa realização profissional (59,9\%), alta exaustão emocional (42,2\%) e moderada despersonalização (58,7\%). A dimensão exaustão emocional foi a que apresentou maior percentual no nível alto, quando comparada com a despersonalização (27,5\%) e a realização profissional (2,8\%). 
Figura 1 - Dimensões do Maslach Burnout Inventory (MBI) de profissionais de enfermagem de uma cidade do oeste Paraense (2021).

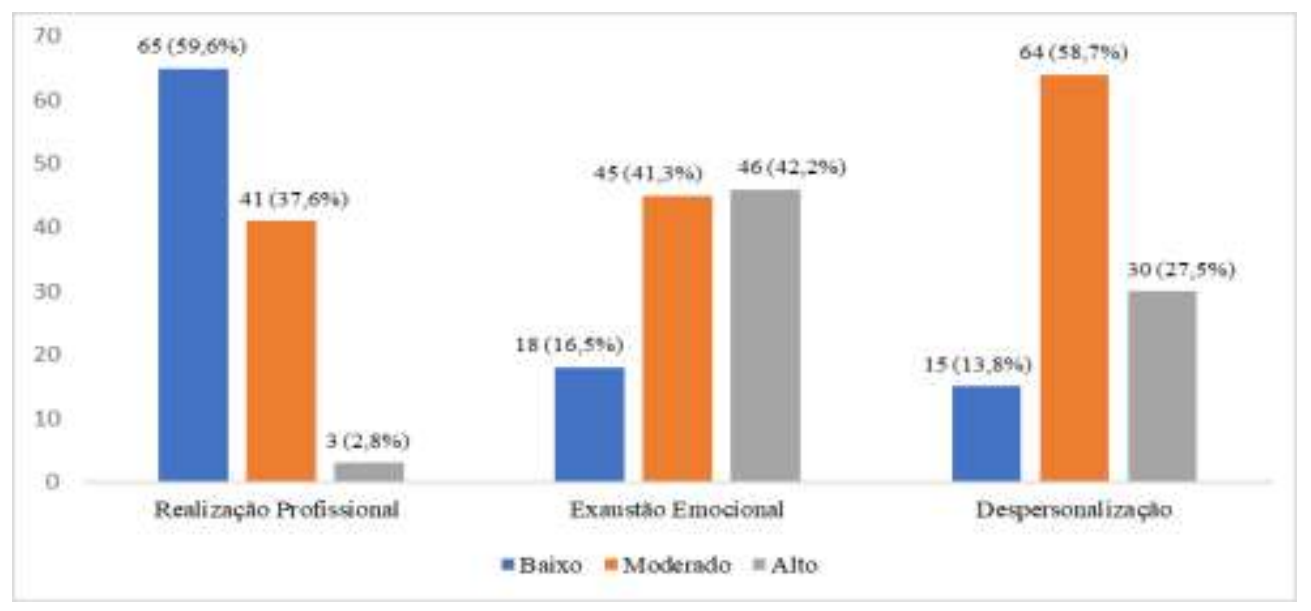

Fonte: Dados da pesquisa.

A associação das dimensões do MBI com dados sociodemográficos, hábitos de saúde e religião e dados laborais são apresentadas nas Tabelas 3, 4 e 5, respectivamente. Não foram identificadas diferenças estatísticas nos dados sociodemográficos (Tabela 3). Na associação com hábitos de saúde e religião, foi verificado que a maioria que não possui religião (92,3\%), apresenta baixa realização profissional $(\mathrm{p}=0,03)$ e alta despersonalização $(61,5 \%)(\mathrm{p}=0,01)$. Alto nível de exaustão profissional foi encontrado nos profissionais de enfermagem que já realizaram tratamento para depressão (100\%) e não praticam atividade física $(43,3 \%)(\mathrm{p}=0,05 ; \mathrm{p}=0,01)$ (Tabela 4). A Tabela 5 apresenta a associação das dimensões com os dados laborais dos profissionais de enfermagem. O sentimento em relação ao trabalho e haver sofrido algum tipo de violência nos últimos doze meses apresentou associação estatisticamente significativa com exaustão emocional e despersonalização. Os mais satisfeitos com seu trabalho $(42,9 \%)$ apresentaram o nível mais baixo de exaustão profissional, enquanto os mais insatisfeitos (66,7\%) apresentaram o nível mais alto $(66,7 \%)(\mathrm{p}=0,04)$. Não foram verificados profissionais pouco insatisfeitos, insatisfeitos ou muito insatisfeitos com nível de despersonalização baixo $(\mathrm{p}=0,02)$. Além disso, os que sofreram algum tipo de violência nos últimos doze meses, apresentaram nível alto de exaustão emocional $(52,7 \%)(\mathrm{p}=0,03)$ e apresentaram uma frequência mais elevada de despersonalização considerada moderada $(52,7 \%)$ ou alta $(40 \%)(p=0,01)$. A despersonalização foi moderada e alta $(92,7 \%)$ para aqueles que sofreram algum tipo de violência nos últimos doze meses no trabalho $(\mathrm{p}=0,03)$. 
Research, Society and Development, v. 10, n. 15, e458101519274. 2021

(CC BY 4.0) | ISSN 2525-3409 | DOI: http://dx.doi.org/10.33448/rsd-v10i15.19274

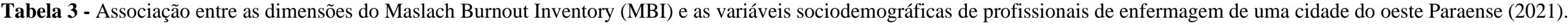

\begin{tabular}{|c|c|c|c|c|c|c|c|c|c|c|c|c|}
\hline \multirow[b]{2}{*}{ Variáveis } & \multicolumn{4}{|c|}{ Realização Profissional } & \multicolumn{4}{|c|}{ Exaustão Emocional } & \multicolumn{4}{|c|}{ Despersonalização } \\
\hline & Baixo & Moderado & Alto & Valor de $\mathrm{p}$ & Baixo & Moderado & Alto & $\begin{array}{c}\text { Valor de } \\
\mathrm{p}\end{array}$ & Baixo & Moderado & Alto & $\begin{array}{c}\text { Valor de } \\
p\end{array}$ \\
\hline Profissão: (b) & & & & 0,65 & & & & 0,26 & & & & 0,54 \\
\hline Técnico de Enfermagem & $35(58,3 \%)$ & $24(40 \%)$ & $1(1,7 \%)$ & & $13(21,7 \%)$ & $24(40 \%)$ & $23(38,3 \%)$ & & $9(15 \%)$ & $37(61,7 \%)$ & & \\
\hline Enfermeiro & $30(61,2 \%)$ & $17(34,7 \%)$ & $2(4,1 \%)$ & & $5(10,2 \%)$ & $21(42,9 \%)$ & $23(46,9 \%)$ & & $6(12,2 \%)$ & $27(55,1 \%)$ & $76(32,7 \%)$ & \\
\hline Sexo: (b) & & & & 0,78 & & & & 0,09 & & & & 0,10 \\
\hline Feminino & $52(57,8 \%)$ & $35(38,9 \%)$ & $3(3,3 \%)$ & & $17(18,9 \%)$ & $39(43,3 \%)$ & $34(37,8 \%)$ & & $13(14,5 \%)$ & $56(62,2 \%)$ & $21(23,3 \%)$ & \\
\hline Masculino & $13(68,4 \%)$ & $6(31,6 \%)$ & $0(0 \%)$ & & $1(5,3 \%)$ & $6(31,6 \%)$ & $12(63,2 \%)$ & & $2(10,5 \%)$ & $8(42,1 \%)$ & $9(47,4 \%)$ & \\
\hline Cor/Etnia: (a) & & & & 0,42 & & & & 0,96 & & & & 0,88 \\
\hline Branca & $3(33,3 \%)$ & $6(66,7 \%)$ & $0(0 \%)$ & & $1(11,1 \%)$ & $3(33,3 \%)$ & $5(55,6 \%)$ & & $2(22,2 \%)$ & $3(33,3 \%)$ & $4(44,5 \%)$ & \\
\hline Parda & $58(62,4 \%)$ & $32(34,4 \%)$ & $3(3,2 \%)$ & & $16(17,2 \%)$ & $39(41,9 \%)$ & $38(40,9 \%)$ & & $11(11,8 \%)$ & $57(61,3 \%)$ & $25(26,9 \%)$ & \\
\hline Negra & $3(50 \%)$ & $3(50 \%)$ & $0(0 \%)$ & & $1(16,7 \%)$ & $3(50 \%)$ & $2(33,3 \%)$ & & $1(16,7 \%)$ & $4(66,7 \%)$ & $1(16,6 \%)$ & \\
\hline Quilombola & $1(100 \%)$ & $0(0 \%)$ & $0(0 \%)$ & & $0(0 \%)$ & $0(0 \%)$ & $1(100 \%)$ & & $1(100 \%)$ & $0(0 \%)$ & $0(0 \%)$ & \\
\hline Estado Civil: (a) & & & & 0,70 & & & & 0,71 & & & & 0,62 \\
\hline Solteira(o) & $29(60,4 \%)$ & $18(37,5 \%)$ & $1(2,1 \%)$ & & $9(18,8 \%)$ & $18(37,5 \%)$ & $21(43,87)$ & & $5(10,4 \%)$ & $30(62,5 \%)$ & $13(27,1 \%)$ & \\
\hline Casada(o) & $17(54,9 \%)$ & $13(41,9 \%)$ & $1(3,2 \%)$ & & $3(9,7 \%)$ & $13(41,9 \%)$ & $15(48,4 \%)$ & & $4(12,9 \%)$ & $19(61,3 \%)$ & $8(25,8 \%)$ & \\
\hline Separada(o) & $1(33,3 \%)$ & $2(66,7 \%)$ & $0(0 \%)$ & & $0(0 \%)$ & $1(33,3 \%)$ & $2(66,7 \%)$ & & $0(0 \%)$ & $3(100 \%)$ & $0(0 \%)$ & \\
\hline Divorciada(o) & $4(100 \%)$ & $0(0 \%)$ & $0(0 \%)$ & & $1(25 \%)$ & $1(25 \%)$ & $2(50 \%)$ & & $1(25 \%)$ & $1(25 \%)$ & $2(50 \%)$ & \\
\hline União Estável & $14(60,9 \%)$ & $8(34,8 \%)$ & $1(4,3 \%)$ & & $5(21,7 \%)$ & $12(52,2 \%)$ & $6(26,1 \%)$ & & $5(21,7 \%)$ & $11(47,8 \%)$ & $7(30,5 \%)$ & \\
\hline Renda mensal individual: a) & & & & 0,06 & & & & 0,30 & & & & 0,12 \\
\hline Até $1 \mathrm{SM}(\mathrm{R} \$ 1.100)$ & $21(77,8 \%)$ & $6(22,2 \%)$ & $0(0 \%)$ & & $7(26 \%)$ & $10(37 \%)$ & $10(37 \%)$ & & $5(18,5 \%)$ & $16(59,3 \%)$ & $6(22,2 \%)$ & \\
\hline Até 2 SM (R \$2.200) & $15(40,5 \%)$ & $19(51,4 \%)$ & $3(8,1 \%)$ & & $8(21,6 \%)$ & $17(45,9 \%)$ & $12(32,5 \%)$ & & $6(16,2 \%)$ & $24(64,9 \%)$ & $7(18,9 \%)$ & \\
\hline Até $3 \mathrm{SM}(\mathrm{R} \$ 3.300)$ & $13(68,4 \%)$ & $6(31,6 \%)$ & $0(0 \%)$ & & $2(10,5 \%)$ & $9(47,4 \%)$ & $8(42,1 \%)$ & & $3(15,7 \%)$ & $12(63,2 \%)$ & $4(21,1 \%)$ & \\
\hline De 3 à 5 SM (R\$3.30 à 5.500) & $13(68,4 \%)$ & $6(31,6 \%)$ & $0(0 \%)$ & & $1(5,3 \%)$ & $7(36,8 \%)$ & $11(57,9 \%)$ & & $1(5,3 \%)$ & $7(36,8 \%)$ & $11(57,9 \%)$ & \\
\hline Acima de 5 SM $(\mathrm{R} \$ 5,500)$ & $3(42,9 \%)$ & $4(57,1 \%)$ & $0(0 \%)$ & & $0(0 \%)$ & $2(28,6 \%)$ & $5(71,4 \%)$ & & $0(0 \%)$ & $5(71,4 \%)$ & $2(28,6 \%)$ & \\
\hline Escolaridade: (a) & & & & 0,49 & & & & 0,16 & & & & 0,81 \\
\hline Médio Completo & $20(69 \%)$ & $9(31 \%)$ & $0(0 \%)$ & & $6(20,7 \%)$ & $13(44,8 \%)$ & $10(34,5 \%)$ & & $5(17,2 \%)$ & $17(58,6 \%)$ & $7(24,2 \%)$ & \\
\hline Superior Incompleto & $9(60 \%)$ & $6(40 \%)$ & $0(0 \%)$ & & $3(20 \%)$ & $7(46,7 \%)$ & $5(33,3 \%)$ & & $2(13,3 \%)$ & $10(66,7 \%)$ & $3(20 \%)$ & \\
\hline Superior Completo & $13(65 \%)$ & $6(30 \%)$ & $1(5 \%)$ & & $7(35 \%)$ & $5(25 \%)$ & $8(40 \%)$ & & $4(20 \%)$ & $11(55 \%)$ & $5(25 \%)$ & \\
\hline Especialização & $23(53,4 \%)$ & $18(41,9 \%)$ & $2(4,7 \%)$ & & $2(4,7 \%)$ & $19(44,2 \%)$ & $22(51,1 \%)$ & & $4(9,3 \%)$ & $24(55,8 \%)$ & $15(34,9 \%)$ & \\
\hline Mestrado & $0(0 \%)$ & $2(100 \%)$ & $0(0 \%)$ & & $0(0 \%)$ & $1(50 \%)$ & $1(50 \%)$ & & $0(0 \%)$ & $2(100 \%)$ & $0(0 \%)$ & \\
\hline
\end{tabular}

Resultados expressos através de análises de frequência. SM-Salário-Mínimo.

(a) Teste Qui Quadrado (b) - Teste Exato de Fischer

Fonte: Dados da pesquisa. 
Research, Society and Development, v. 10, n. 15, e458101519274. 2021

(CC BY 4.0) | ISSN 2525-3409 | DOI: http://dx.doi.org/10.33448/rsd-v10i15.19274

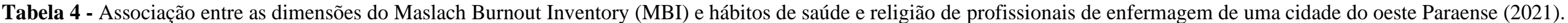

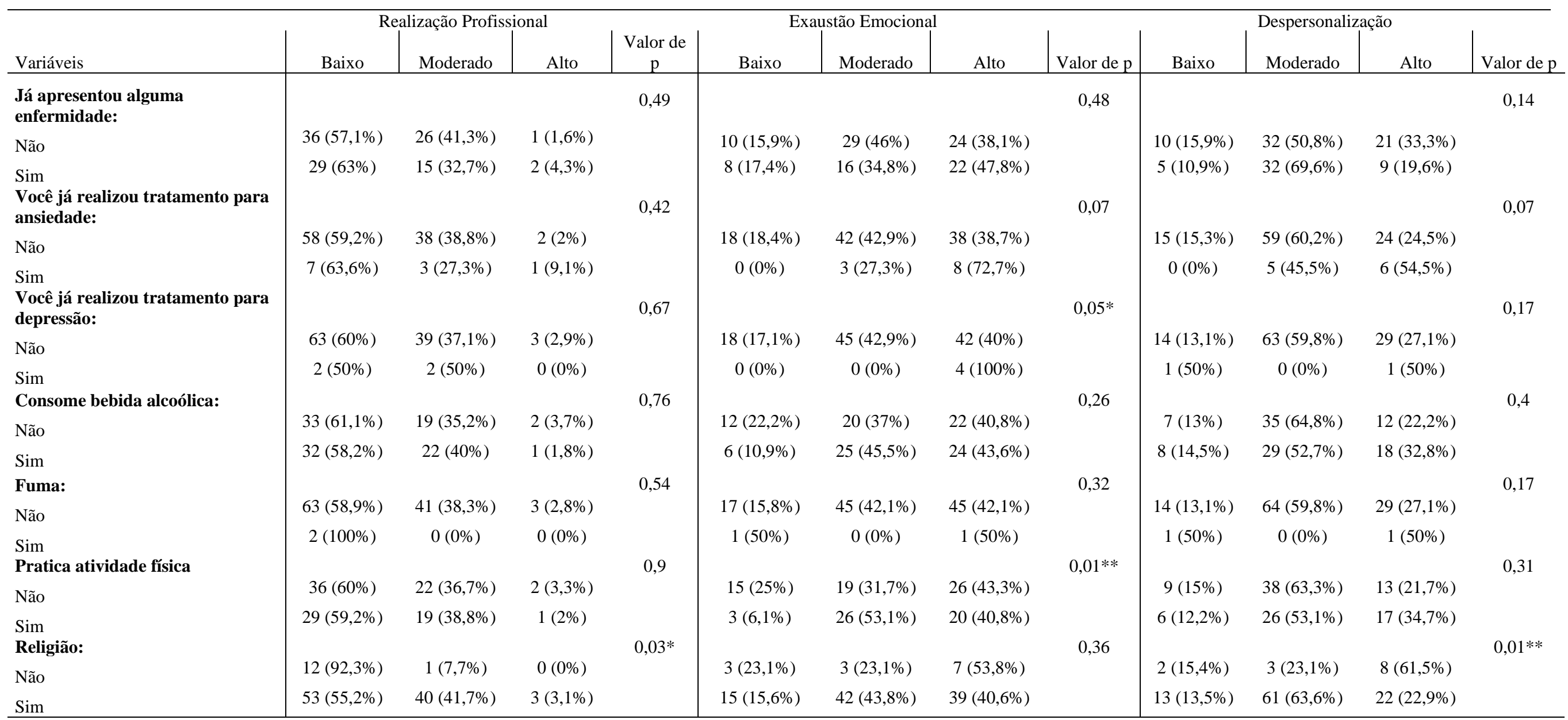

Resultados expressos através de análises de frequência.

** Significativo ao nível de 0,01* Significativo ao nível de 0,05 - Teste Exato de Fischer

Fonte: Dados da pesquisa 
Tabela 5 - Associação entre as dimensões do Maslach Burnout Inventory (MBI) e dados laborais profissionais de enfermagem de uma cidade do oeste Paraense (2021).

\begin{tabular}{|c|c|c|c|c|c|c|c|c|c|c|c|c|}
\hline \multirow[b]{2}{*}{ Variáveis } & \multicolumn{3}{|c|}{ Realização Profissional } & \multirow[b]{2}{*}{$\begin{array}{l}\text { Valor de } \\
\mathrm{p}\end{array}$} & \multicolumn{4}{|c|}{ Exaustão Emocional } & \multicolumn{4}{|c|}{ Despersonalização } \\
\hline & Baixo & Moderado & Alto & & Baixo & Moderado & Alto & $\begin{array}{l}\text { Valor de } \\
\text { p }\end{array}$ & Baixo & Moderado & Alto & $\begin{array}{l}\text { Valor de } \\
\mathrm{p}\end{array}$ \\
\hline $\begin{array}{l}\text { Você desenvolve outra atividade } \\
\text { remunerada: (a) }\end{array}$ & & & & 0,34 & & & & 0,19 & & & & 0,12 \\
\hline Não & $48(61,5 \%)$ & $29(37,2 \%)$ & $1(1,3 \%)$ & & $16(20,5 \%)$ & $30(38,5 \%)$ & $32(41 \%)$ & & $14(17,9 \%)$ & $43(55,2 \%)$ & $21(26,9 \%)$ & \\
\hline Sim & $17(54,8 \%)$ & $12(38,7 \%)$ & $2(6,5 \%)$ & & $2(6,5 \%)$ & $15(48,4 \%)$ & $14(45,1 \%)$ & & $1(3,2 \%)$ & $21(67,8 \%)$ & $9(29 \%)$ & \\
\hline $\begin{array}{l}\text { Há quanto tempo você trabalha como } \\
\text { enfermeiro ou técnico de enfermagem: (b) }\end{array}$ & & & & 0,42 & & & & 0,26 & & & & 0,7 \\
\hline entre 1 e 2 anos & $10(62,5 \%)$ & $6(37,5 \%)$ & $0(0 \%)$ & & $3(18,8 \%)$ & $10(62,5 \%)$ & $3(18,8 \%)$ & & $3(18,8 \%)$ & $10(62,5 \%)$ & $3(18,7 \%)$ & \\
\hline entre 2 e- 4 anos & $10(58,1 \%)$ & $6(35,3 \%)$ & $1(5,9 \%)$ & & $3(17,6 \%)$ & $9(52,9 \%)$ & $5(29,5 \%)$ & & $2(11,8 \%)$ & $12(70,6 \%)$ & $3(17,6 \%)$ & \\
\hline entre 4 e 6 anos & $14(73,7 \%)$ & $5(26,3 \%)$ & $0(0 \%)$ & & $2(10,5 \%)$ & $8(42,1 \%)$ & $9(47,4 \%)$ & & $2(10,5 \%)$ & $10(52,6 \%)$ & $7(36,9 \%)$ & \\
\hline entre 6 e 8 anos & $4(40 \%)$ & $5(50 \%)$ & $1(10 \%)$ & & $1(10 \%)$ & $5(50 \%)$ & $4(40 \%)$ & & $1(10 \%)$ & $6(60 \%)$ & $3(30 \%)$ & \\
\hline Mais de oito anos & $22(57,9 \%)$ & $16(42,1 \%)$ & $0(0 \%)$ & & $6(15,8 \%)$ & $10(26,3 \%)$ & $22(57,9 \%)$ & & $4(10,5 \%)$ & $21(55,3 \%)$ & $13(34,2 \%)$ & \\
\hline $\begin{array}{l}\text { Qual a seu sentimento em relação ao seu } \\
\text { trabalho:(b) }\end{array}$ & & & & 0,93 & & & & $0,04 *$ & & & & $0,02 *$ \\
\hline Muito satisfeito & $9(64,3 \%)$ & $5(35,7 \%)$ & $0(0 \%)$ & & $6(42,9 \%)$ & $5(35,7 \%)$ & $3(21,4 \%)$ & & $4(28,6 \%)$ & $8(57,2 \%)$ & $2(14,3 \%)$ & \\
\hline Satisfeito & $28(56 \%)$ & $19(38 \%)$ & $3(6 \%)$ & & $10(20 \%)$ & $25(25 \%)$ & $15(30 \%)$ & & $11(22 \%)$ & $30(60 \%)$ & $9(18 \%)$ & \\
\hline Pouco Satisfeito & $21(63,6 \%)$ & $12(36,4 \%)$ & $0(0 \%)$ & & $1(3 \%)$ & $11(33,3 \%)$ & $21(63,7 \%)$ & & $0(0 \%)$ & $20(60,6 \%)$ & $13(39,4 \%)$ & \\
\hline Muito insatisfeito & $2(66,7 \%)$ & $1(33,3 \%)$ & $0(0 \%)$ & & $1(33,3 \%)$ & $0(0 \%)$ & $2(66,7 \%)$ & & $0(0 \%)$ & $1(33,3 \%)$ & $2(66,7 \%)$ & \\
\hline Ha quanto tempo não tira Férias: (b) & & & & 0,86 & & & & 0,19 & & & & 0,5 \\
\hline Menos de um ano & $11(57,9 \%)$ & $7(36,8 \%)$ & $1(5,3 \%)$ & & $3(15,8 \%)$ & $4(21,1 \%)$ & $12(63,1 \%)$ & & $3(15,8 \%)$ & $8(42,1 \%)$ & $8(42,1 \%)$ & \\
\hline Um ano & $4(66,7 \%)$ & $2(33,3 \%)$ & $0(0 \%)$ & & $0(0 \%)$ & $3(50 \%)$ & $3(50 \%)$ & & $1(16,7 \%)$ & $3(50 \%)$ & $2(33,3 \%)$ & \\
\hline Mais de um ano. & $50(59,5 \%)$ & $32(38,1 \%)$ & $2(2,4 \%)$ & & $15(17,9 \%)$ & $38(45,2 \%)$ & $31(36,9 \%)$ & & $11(13,1 \%)$ & $53(63,1 \%)$ & $20(23,8 \%)$ & \\
\hline $\begin{array}{l}\text { Em 2020, você esteve ausente por } 2 \text { dias } \\
\text { ou mais no trabalho: (a) }\end{array}$ & & & & 0,56 & & & & 0,12 & & & & 0,5 \\
\hline Não & $22(66,7 \%)$ & $10(30,3 \%)$ & $1(3 \%)$ & & $9(27,3 \%)$ & $11(33,3 \%)$ & $13(39,4 \%)$ & & $6(18,2 \%)$ & $20(60,6 \%)$ & $7(21,2 \%)$ & \\
\hline $\operatorname{Sim}$ & $43(56,6 \%)$ & $3(40,8 \%)$ & $2(2,6 \%)$ & & $9(11,8 \%)$ & $34(44,7 \%)$ & $33(43,5 \%)$ & & $9(11,8 \%)$ & $44(57,9 \%)$ & $23(30,3 \%)$ & \\
\hline $\begin{array}{l}\text { Nos últimos } 12 \text { meses, você sofreu algum } \\
\text { tipo de violência no seu trabalho: (a) }\end{array}$ & & & & 0,52 & & & & $0,03 *$ & & & & $0,01 * *$ \\
\hline Não & $35(64,8 \%)$ & $18(33,3 \%)$ & $1(1,9 \%)$ & & $13(24,1 \%)$ & $24(44,4 \%)$ & $17(31,5 \%)$ & & $11(20,4 \%)$ & $35(64,8 \%)$ & $8(14,8 \%)$ & \\
\hline Sim & $30(54,6 \%)$ & $23(41,8 \%)$ & $2(3,6 \%)$ & & $5(9,1 \%)$ & $21(38,2 \%)$ & $29(52,7 \%)$ & & $4(7,3 \%)$ & $29(52,7 \%)$ & $22(40 \%)$ & \\
\hline $\begin{array}{l}\text { Você acha que é afetado pela síndrome de } \\
\text { Burnout (a) }\end{array}$ & & & & 0,24 & & & & 0,24 & & & & $0,03 *$ \\
\hline Não & $54(62,8 \%)$ & $29(33,7 \%)$ & $3(3,5 \%)$ & & $16(18,6 \%)$ & $37(43 \%)$ & $33(38,4 \%)$ & & $14(16,3 \%)$ & $53(61,6 \%)$ & $19(22,1 \%)$ & \\
\hline Sim & $11(47,8 \%)$ & $12(52,2 \%)$ & $0(0 \%)$ & & $2(8,7 \%)$ & $8(34,8 \%)$ & $13(56,5 \%)$ & & $1(4,3 \%)$ & $11(47,8 \%)$ & $11(47,9 \%)$ & \\
\hline
\end{tabular}

Resultados expressos através de análises de frequência

** Significativo ao nível de 0,01. * Significativo ao nível de 0,05 - (a) Teste Qui Quadrado (b) - Teste Exato de Fischer

Fonte: Dados da pesquisa 


\section{Discussão}

Esta pesquisa identificou que, durante a pandemia, a SB estava presente em 12,8\% dos profissionais de enfermagem de um município do oeste do Pará. Em abril de 2021, quando foi realizada, o município apresentava uma queda na internação nos leitos clínicos para Covid-19, diminuindo de $64 \%$ no início do mês para $41 \%$ ao final do mês, da mesma maneira para ocupação de leitos em UTI, que de $85 \%$ passou para $31 \%$. Estes índices decrescentes podem ter influenciado nos resultados deste estudo. Resultado superior a esta pesquisa foi encontrado em um trabalho que identificou a prevalência de 25,5\% de SB em técnicos de enfermagem de UTI durante a pandemia, em 2020, em uma cidade no norte de Minas Gerais (Freitas et al., 2021). Antes da pandemia a prevalência de SB variou entre 4,7\% e 20,83,3\% (Borges et al., 2021, Carvalho, Silva, Bachur, Mesquita, \& França-Botelho, 2016, Galindo, Feliciano, Lima, \& Souza, 2012, Tavares et al., 2014, Vasconcelos \& De Martino, 2017). Na China, o primeiro estudo realizado com enfermeiros da linha de frente ao combate da Covid-19 em Wuhan identificou níveis moderados de SB (Hu et al. 2020), assim como na Turquia, durante o início da pandemia, altos níveis de SB foram encontrados em enfermeiros (Murat, Köse, \& Savaşer, 2021).

Nesse estudo, o índice mais alto entre os profissionais de enfermagem foi a baixa realização profissional (59,9\%), seguido da alta exaustão emocional (42,2\%) e moderada despersonalização (58,7\%). Estudo realizado com enfermeiros da linha de frente de Covid-19 em Wuhan, na China, encontrou dados semelhantes, 41,5\% apresentaram alta exaustão emocional, 27,6\% alta despersonalização e 38,3\% baixa realização profissional (Hu et al., 2020). No Brasil, pesquisa realizada com profissionais de enfermagem no munícipio de Campina Grande/Paraíba identificou que a maioria apresentava baixa realização profissional e média despersonalização (Ribeiro et al., 2021).

Os profissionais dessa pesquisa estavam atuando na linha de frente ao combate da pandemia, o que pode ter acentuado os altos níveis de exaustão emocional e baixa realização profissional. Enfrentavam permanentemente o risco da própria contaminação ou de um ente querido. A ideia de culpa relacionadas às famílias, seja pela distância do isolamento ou pela proximidade na convivência, que aumenta a chance de contágio, foi relatada por profissionais da saúde durante a pandemia (Horta et al., 2021). Em enfermeiros de hospitais na Espanha foi identificada uma correlação positiva da sobrecarga de trabalho com a SB durante a pandemia, e a percepção da ameaça de Covid-19 ajudou a explicar o grau da SB (Manzano García \& Ayala Calvo, 2021). Ainda, profissionais de saúde (médicos e enfermeiros) que cuidavam de pacientes de Covid-19 apresentaram níveis mais altos de SB (Ruiz-Fernandez et al., 2020).

A enfermagem possui muitas atribuições, como planejamento, gerenciamento, coordenação e avaliação das ações individuais e coletivas das equipes de saúde em que estão inseridas. É considerada uma profissão com alto estresse ocupacional, que pode afetar o psicológico durante suas relações de trabalho (Abreu et al., 2020). Neste estudo foi identificada associação estatística entre as dimensões da SB, exaustão emocional e despersonalização e dados de trabalho destes profissionais $(\mathrm{p} \leq 0,05)$. Profissionais que estavam pouco satisfeitos, insatisfeitos ou muito insatisfeitos com o trabalho apresentaram níveis mais altos de exaustão emocional $(\mathrm{p}=0,04)$ e níveis moderados e altos de despersonalização ( $\mathrm{p}=0,02)$. A relação entre as dimensões da SB e as variáveis de trabalho de profissionais de enfermagem de um hospital municipal de emergência da cidade do Rio de Janeiro/RJ, mostrou que o esgotamento emocional é maior entre aqueles que percebem o ambiente de trabalho como ruim/muito ruim em comparação com aqueles que consideram regular, bom/muito bom (Soares et al., 2021). No desenvolvimento da SB entre profissionais de enfermagem, a exaustão emocional tem sido caracterizada como central e a despersonalização frequentemente acompanha este sentimento (Dutra et al., 2019). A carga horária excessiva e a insatisfação profissional foram associadas a SB em enfermeiros da Rede de Atenção Primária à Saúde do município de Aracaju/SE (Silva et al., 2015). Entre profissionais da saúde de um hospital de grande porte de Belo Horizonte/MG foi encontrada correlação positiva entre exaustão emocional e insatisfação com o trabalho, mostrando-se como o primeiro marcador identificável da SB (Rocha et al., 2019). 
A pandemia pode ter agravado este quadro de sobrecarga laboral vivenciado pelos profissionais de enfermagem dessa pesquisa. Na Itália, os enfermeiros foram mais suscetíveis a SB, principalmente em função do cargo que ocupam na organização de trabalho e das atribuições que geralmente lhes são atribuídas. Durante a pandemia de Covid-19, os enfermeiros da linha de frente enfrentaram desafios repentinos e dramáticos no local de trabalho em termos de aumento da carga de trabalho, reatribuição/redistribuição para outras funções ou funções, ameaça de infecção, eventos traumáticos relacionados a pandemia e frustração com a morte de pacientes que eles se importam. Essa sobrecarga de trabalho pode ter contribuído para o desgaste do enfermeiro ao esgotar sua capacidade emocional para atender às demandas da emergência pandêmica (Lasalvia et al., 2021). Os resultados de uma pesquisa mista realizada com profissionais da saúde no Rio Grande do Sul (76\% enfermeiros) sugerem que sobrecarga na linha de frente da pandemia foi a principal fonte de sofrimento. Os profissionais relataram dificuldade de lidar com as situações adversas, cansaço e pressão maior que o habitual. Os longos plantões foram agravados pela dificuldade de realizar intervalos, devido a paramentação, que precisa ser desfeita a cada saída da área reservada a pacientes de Covid-19 (Horta et al., 2021). Em Singapura, durante a pandemia os limiares da SB em desengajamento e exaustão foram alcançados por 79,7\% e 75,3\% dos profissionais da saúde, respectivamente. Turnos maiores que oito horas também foram associados a escores mais altos da SB (Tan et al, 2020).

Nessa pesquisa, a maioria dos profissionais que se perceberam afetados pela SB apresentaram níveis moderados e altos de despersonalização $(\mathrm{p}=0,03)$. Também, os participantes que relataram ter sofrido algum tipo de violência nos últimos 12 meses no trabalho apresentaram uma frequência mais elevada de exaustão emocional e despersonalização, comparando com os que não sofreram $(\mathrm{p} \leq 0,03)$. Uma pesquisa realizada por Lima e Sousa (2015) apontou que a incidência de violência psicológica no ambiente hospitalar é comum, no entanto, ocorre de certa forma velada, devido ao medo, a vergonha da situação vivida, a falta de tempo, bem como o não reconhecimento pelos empregados e empregadores. A violência sofrida no ambiente de trabalho por enfermeiros da Atenção Básica foi associada a SB na Bahia (Merces et al., 2020).

Com relação aos hábitos de saúde, todos os profissionais que realizaram tratamento para depressão apresentaram altos níveis de exaustão emocional, comparado com $40 \%$ dos que não realizaram $(\mathrm{p}=0,05)$. Profissionais de enfermagem que desenvolveram o quadro de depressão têm mais probabilidade de manifestarem a SB (Santos et al., 2021). Considerando a pandemia, um estudo de larga escala realizado na China com enfermeiros identificou que aqueles que estavam envolvidos com tratamento de pacientes com covid apresentaram escores mais altos nos desfechos de saúde mental (Chen et al., 2021). Da mesma maneira, na Itália, a SB foi mais frequente em profissionais da saúde que estiveram em tratamento psicológico (Lasalvia et al., 2021). Uma maior prevalência de sintomas psicológicos, como insônia severa, ansiedade, depressão, somatização e sintoma obsessivo-compulsivo foram encontrados em profissionais da saúde durante a pandemia de Covid-19, na China (Zhang et al., 2020). Foi identificado que pessoas com transtornos de humor ou relacionados à ansiedade foram mais afetadas negativamente pela Covid-19 em comparação com aqueles sem transtorno de saúde mental (Asmundson et al., 2020). Na China, profissionais da saúde da linha de frente do combate a Covid-19 relataram apresentar problemas psicológicos, principalmente enfermeiras mulheres (Lai et al., 2020). Estresse, sobrecarga de trabalho, esgotamento físico, depressão e interação social comprometida foram os fatores de risco identificados para profissionais da saúde durante a pandemia de Covid-19, segundo uma revisão de literatura (Borges et al., 2021).

A maioria dos participantes que praticavam alguma atividade física apresentou níveis moderados de exaustão emocional, enquanto os que não praticavam apresentaram níveis altos $(\mathrm{p}=0,01)$. Pesquisa realizada no estado da Bahia também identificou, entre profissionais de enfermagem da Atenção Primária, que a falta de uma rotina de atividade física foi um fator relevante para ocorrer a SB (Merces et al., 2020).

Com relação a religião, $61,5 \%$ dos profissionais de enfermagem que afirmaram não possuir uma religião apresentaram alto nível de despersonalização, enquanto $63,6 \%$ dos que possuíam apresentaram nível moderado (p=0,01). A justificativa pode 
ser devido ao fato de que existe uma espiritualidade inconsciente em todo o ser humano que acaba afetando as mais autênticas tomadas de decisões de sua existência e são produzidas no inconsciente humano (Silva et al., 2016).

Os profissionais de saúde costumam passar por estresse ocupacional que leva ao esgotamento, podendo ser agravado durante a pandemia. É essencial melhorar as medidas organizacionais para criar um impacto duradouro na cultura de trabalho ao lado de intervenções interpessoais e lidar com o estresse no local de trabalho. Os legisladores e profissionais de saúde devem adotar intervenções e desenvolver abordagens específicas ao contexto que promovam um ambiente de trabalho saudável, abordando questões éticas e prevenindo o esgotamento entre os profissionais de saúde durante a pandemia de Covid19 (Sultana et al., 2020). Para minimizar as implicações negativas e promover a saúde mental, intervenções psicológicas podem ser realizadas não somente durante a pandemia, mas também em momentos posteriores, quando os profissionais necessitarão lidar com as perdas e transformações (Schmidt, Crepaldi, Bolze, Neiva-Silva \& Demenech, 2020).

Como limitações desta pesquisa pode-se citar a natureza transversal, que não permite a determinação de relações casuais e reflete apenas o momento da coleta. Além disso, características psicológicas individuais não foram consideradas entre os possíveis preditores. Os dados encontrados deste estudo consideraram somente a prevalência da SB em profissionais de enfermagem, não levando em consideração as consequências ocasionadas pela manifestação dessa síndrome. Para tanto, a fim de verificar tais consequências será necessária a realização de novos estudos para que essa questão seja verificada e aprofundada, auxiliando na construção de protocolos e diretrizes para o enfrentamento.

\section{Considerações Finais}

Os profissionais de saúde, em especial os de enfermagem, lidam, em seu cotidiano, com diversas situações complexas que demandam muito de sua saúde. A SB aparece quando o indivíduo está exposto a situações crônicas de estresse no trabalho e pode prejudicar diversos fatores na vida do indivíduo, tais como profissional, pessoal, familiar e levando-o a um declínio de capacidade no trabalho. Durante a pandemia de Covid-19 foi identificada uma prevalência de 12,8\% da SB em profissionais de enfermagem em um município de Oeste Paraense, sendo maior em enfermeiros que técnicos de enfermagem. Nesse sentido, é importante o desenvolvimento de ações voltadas para a promoção da saúde e bem-estar desses profissionais.

\section{Referências}

Abreu, K. L., Stoll, I., Ramos, L. S., Baumgardt, R. A \& Kristensen, C. H. (2020). Estresse ocupacional e síndrome de Burnout no exercício profissional da psicologia. Psicologia: Ciência e Profissão, 22(2), 22-29. https://doi.org/10.1590/S1414-98932002000200004

ANAMT - Associação Nacional de Medicina do Trabalhado (2018). $30 \%$ dos trabalhadores brasileiros sofrem com síndrome de Burnout. https://www.anamt.org.br/portal/2018/12/12/30-dos-trabalhadores-brasileiros-sofrem-com-a-sindrome-de-burnout/.

Asmundson, G., Paluszek, M. M., Landry, C. A., Rachor, G. S., McKay, D., \& Taylor, S. (2020). Do pre-existing anxiety-related and mood disorders differentially impact COVID-19 stress responses and coping?. Journal of anxiety disorders, 74, 102271. https://doi.org/10.1016/j.janxdis.2020.102271

Backes, M., Higashi, G., Damiani, P., Mendes, J. S., Sampaio, L. S., \& Soares, G. L. (2021). Condições de trabalho dos profissionais de enfermagem no enfrentamento da pandemia da covid-19. Revista gaucha de enfermagem, 42(spe), e20200339. https://doi.org/10.1590/1983-1447.2021.20200339

Borges, E. M. das N., Queirós, C. M. L., Abreu, M. da S. N. de, Mosteiro-Diaz, M. P., Baldonedo-Mosteiro, M., Baptista, P. C. P., Felli, V. E. A., Almeida, M. C. dos S., \& Silva, S. M. (2021). Burnout entre enfermeiros: um estudo multicêntrico comparativo. Revista Latino-Americana De Enfermagem, 29 , e3432. https://doi.org/10.1590/1518-8345.4320.3432

Borges, F. E. de, Borges Aragão, D., Borges, F. E., Borges, F. E., Sousa, A. S., \& Machado, A. L. (2021). Fatores de risco para a Síndrome de Burnout em profissionais da saúde durante a pandemia de COVID-19. Revista Enfermagem Atual In Derme, 95(33), e-021006. https://doi.org/10.31011/reaid-2020-v.94n.32-art.835

Brasil. Lei no 13.979 de 6 de fevereiro de 2020. Dispõe sobre as medidas para enfrentamento da emergência de saúde pública de importância internacional decorrente do coronavírus responsável pelo surto de 2019. Diário Oficial da União, Brasília, 1, 7 fev. 2020. <http:// www.planalto.gov.br/ccivil_03/_ato20192022/2020/lei/113979.htm>.

Carvalho, D. de, Silva, N., Bachur, J. A., Mesquita, J. L. F., \& França-Botelho, A. do C. (2016). Síndrome de burnout em profissionais da área da saúde atuantes em dois municípios do interior de Minas Gerais - Brasil. Revista Contexto \&Amp; Saúde, 16(31), 139-148. https://doi.org/10.21527/21767114.2016.31.139-148 
Chen, R., Sun, C., Chen, J. J., Jen, H. J., Kang, X. L., Kao, C. C., \& Chou, K. R. (2021). A Large-Scale Survey on Trauma, Burnout, and Posttraumatic Growth among Nurses during the COVID-19 Pandemic. International journal of mental health nursing, 30(1), 102-116. https://doi.org/10.1111/inm.12796

Dutra, H.S., Aparecida Lopes Gomes, P., Nereu Garcia, R., Ceretta Oliveira, H., Carvalho de Freitas, S., \& de Brito Guirardello, E. (2019). Burnout entre profissionais de enfermagem em hospitais no Brasil. Revista Cuidarte, 10(1). https://doi.org/10.15649/cuidarte.v10i1.585

Freitas, R. F., Barros, I. M. D., Miranda, M. A. F., Freitas, T. F., Rocha, J. S. B., \& Lessa, A. D. C. (2021). Preditores da síndrome de Burnout em técnicos de enfermagem de unidade de terapia intensiva durante a pandemia da COVID-19. Jornal Brasileiro de Psiquiatria, 70, 12-20. https://doi.org/10.1590/00472085000000313

Galindo, R. H., Feliciano, K. V. D. O., Lima, R. A. D. S., \& Souza, A. I. D. (2012). Síndrome de Burnout entre enfermeiros de um hospital geral da cidade do Recife. Revista da Escola de Enfermagem da USP, 46, 420-427. https://doi.org/10.1590/S0080-62342012000200021

Horta, R. L., Camargo, E. G., Barbosa, M. L. L., Lantin, P. J. S., Sette, T. G., Lucini, T. C. G., ... \& Lutzky, B. A. (2021). O estresse e a saúde mental de profissionais da linha de frente da COVID-19 em hospital geral. Jornal Brasileiro de Psiquiatria, 70, 30-38. https://doi.org/10.1590/0047-2085000000316

Hu, D., Kong, Y., Li, W., Han, Q., Zhang, X., Zhu, L. X., Wan, S. W., Liu, Z., Shen, Q., Yang, J., He, H. G., \& Zhu, J. (2020). Frontline nurses' burnout, anxiety, depression, and fear statuses and their associated factors during the COVID-19 outbreak in Wuhan, China: A large-scale cross-sectional study. EClinicalMedicine, 24, 100424. https://doi.org/10.1016/j.eclinm.2020.100424

Lai, J., Ma, S., Wang, Y., Cai, Z., Hu, J., Wei, N., Wu, J., Du, H., Chen, T., Li, R., Tan, H., Kang, L., Yao, L., Huang, M., Wang, H., Wang, G., Liu, Z., \& Hu, S. (2020). Factors Associated With Mental Health Outcomes Among Health Care Workers Exposed to Coronavirus Disease 2019. JAMA network open, 3(3), e203976. https://doi.org/10.1001/jamanetworkopen.2020.3976

Lasalvia, A., Amaddeo, F., Porru, S., Carta, A., Tardivo, S., Bovo, C., Ruggeri, M., \& Bonetto, C. (2021). Levels of burn-out among healthcare workers during the COVID-19 pandemic and their associated factors: a cross-sectional study in a tertiary hospital of a highly burdened area of north-east Italy. BMJ open, 11(1), e045127. https://doi.org/10.1136/bmjopen-2020-045127

Lautert, L. (1995). O desgaste profissional do enfermeiro. Tese doutorado, Faculdade de Psicologia, Universidade Pontifícia de Salamanca.

Lima, G. H. A., \& Sousa, S. M. A. (2015) Violência psicológica no trabalho da enfermagem. Revista Brasileira de Enfermagem, 68(5), 817-823 Disponível em: https://www.scielo.br/j/reben/a/Gb9KDbGB75rkkCz5NLYpMZn/abstract/?lang=pt

Manzano García, G., \& Ayala Calvo, J. C. (2021). The threat of COVID-19 and its influence on nursing staff burnout. Journal of advanced nursing, 77(2), 832-844. https://doi.org/10.1111/jan.14642

Maslach, C., \& Jackson, S. (1986). Maslach Burnout Inventary. Manual. University of California. Consulting Psychologists.

Mehta, C. R. \& Patel N. R. A. (1980) Network algorithm for the exact treatment of the 2xk contingency table. Communications in Statistics. 78(382), 427-434.

Merces, M. C., Coelho, J. M. F., Lago, S. B., da Silva, D. A. R., de Souza, M. C., Maciel, D. M. C., Marinho, M. C. G., França, S. L. G., Santos, K. A., Castro, J. O., Santos, M. P. S., Coutinho, M. O., Maciel, R. R. B. T., Camelier, F. W. R., Lua, I., Servo, M. L. S., Sobrinho, C. L. N. , Barreto, F. L., E Silva, D. S, \& Passos, K. G.. (2020). Prevalence and factors associated with burnout syndrome among primary health care nursing professionals: A cross-sectional study. International Journal of Environmental Research and Public Health, 17(2). https://doi.org/10.3390/ijerph17020474

Murat, M., Köse, S., \& Savaşer, S. (2021). Determination of stress, depression and burnout levels of front-line nurses during the COVID-19 pandemic. International journal of mental health nursing, 30(2), 533-543. https://doi.org/10.1111/inm.12818

Ribeiro, E. K. D. A., Santos, R. C. D., Araújo-Monteiro, G. K. N. D., Brandão, B. M. L. D. S., Silva, J. C. D., \& Souto, R. Q. (2021). Influência da síndrome de burnout na qualidade de vida de profissionais da enfermagem: estudo quantitativo. Revista Brasileira de Enfermagem,74, e20200298. https://doi.org/10.1590/0034-7167-2020-0298

Rocha, L. J., Cortes, M. C. J. W., Dias, E. C., Fernandes, F. M., \& Gontijo, E. D. (2019). Esgotamento profissional e satisfação no trabalho em trabalhadores do setor de emergência e terapia intensiva em hospital público. Revista Brasileira de medicina do trabalho, 17(3), 300-12. https://doi.org/10.5327/Z1679443520190404.

Ross J. (2020). The Exacerbation of Burnout During COVID-19: A Major Concern for Nurse Safety. Journal of perianesthesia nursing : official journal of the American Society of PeriAnesthesia Nurses, 35(4), 439-440. https://doi.org/10.1016/j.jopan.2020.04.001

Ruiz-Fernández, M. D., Ramos-Pichardo, J. D., Ibáñez-Masero, O., Cabrera-Troya, J., Carmona-Rega, M. I., \& Ortega-Galán, Á. M. (2020). Compassion fatigue, burnout, compassion satisfaction and perceived stress in healthcare professionals during the COVID-19 health crisis in Spain. Journal of clinical nursing, 29(21-22), 4321-4330. https://doi.org/10.1111/jocn.15469

Santos, K. M. R., Galvão, M. H. R., Gomes, S. M., Souza, T. A. Medeiros, A. A., \& Barbosa, I. R. (2021). Depressão e ansiedade em profissionais de enfermagem durante a pandemia da covid-19, Escola Anna Nery, Revista de enfermagem, 25(spe), e20200370 DOI: https://doi.org/10.1590/2177-9465-EAN2020-0370

Schmidt, B., Crepaldi, M. A., Bolze, S. D. A., Neiva-Silva, L., \& Demenech, L. M. (2020). Saúde mental e intervenções psicológicas diante da pandemia do novo coronavírus (COVID-19). Estudos de Psicologia (Campinas), 37, e200063. http://dx.doi.org/10.1590/1982-0 275202037e200063

Silva, J. B., Aquino, T. A. A., \& Silva, A. F. (2016) As relações entre espiritualidade e cuidado segundo as concepções de estudantes de enfermagem. Revista enfermagem. UFPE online, 10(3),1029-37. https://doi.org/10.5205/reuol.8702-76273-4-SM.1003201612

Silva, S. C. P. S., Nunes, M. A. P., Santana, V. R., Reis, F. P., Neto, J. M., \& Lima, S. O. (2015) A síndrome de Burnout em profissionais da Rede de Atenção Primária à Saúde de Aracaju, Brasil. Ciência \& Saúde Coletiva, 20(10), 3011-3020. https://doi.org/10.1590/1413-812320152010.19912014. 
Research, Society and Development, v. 10, n. 15, e458101519274. 2021

(CC BY 4.0) | ISSN 2525-3409 | DOI: http://dx.doi.org/10.33448/rsd-v10i15.19274

Soares, R. da S.., Teixeira, E. R.., Silva, J. L. L. da., \& Chagas, M. C. (2021). Burnout e fatores associados entre profissionais de enfermagem de hospital municipal. Research, Society and Development, 10(3), e0510312909. https://doi.org/10.33448/rsd-v10i3.12909

Souza, N. V. D. D. O., Carvalho, E. C., Soares, S. S. S., Varella, T. C. M. Y., Pereira, S. R. M., \& Andrade, K. B. S. D. (2021). Trabalho de enfermagem na pandemia da covid-19 e repercussões para a saúde mental dos trabalhadores. Revista Gaúcha de Enfermagem, 42. https://doi.org/10.1590/19831447.2021.20200225

Sultana, A., Sharma, R., Hossain, M. M., Bhattacharya, S., \& Purohit, N. (2020). Burnout among healthcare providers during COVID-19: Challenges and evidence-based interventions. Indian journal of medical ethics, V(4), 1-6. https://doi.org/10.20529/IJME.2020.73

Tan, B., Kanneganti, A., Lim, L., Tan, M., Chua, Y. X., Tan, L., Sia, C. H., Denning, M., Goh, E. T., Purkayastha, S., Kinross, J., Sim, K., Chan, Y. H., \& Ooi, S. (2020). Burnout and Associated Factors Among Health Care Workers in Singapore During the COVID-19 Pandemic. Journal of the American Medical Directors Association, 21(12), 1751-1758.e5. https://doi.org/10.1016/j.jamda.2020.09.035

Tavares, k. F. A., Souza, N. V. O., Silva, L. D., \& Kestenberg, C. C. F. (2014). Ocorrência da síndrome de Burnout em enfermeiros residentes. Acta paulista de enfermagem, 27(3), 260-265. https://doi.org/10.1590/1982-0194201400044.

Trigo, R. T., Teng, C. T., \& Hallak, J. E. C. (2007). Síndrome de Burnout ou estafa profissional e os transtornos psiquiátricos. Revista psiquiatria Clinica, 34(5), 223-233. https://doi.org/10.1590/S0101-60832007000500004.

Vasconcelos, E. M., \& De Martino, M. M. F. (2017). Preditores da síndrome de burnout em enfermeiros de unidade de terapia intensiva, Revista Gaúcha de Enfermagem, 38(4), e65354 https://doi.org/10.1590/1983-1447.2017.04.65354.

Vasconcelos, E. M., Martino M. M. F., \& França, S. P. S. (2018). Burnout and depressive symptoms in intensive care nurses: relationship analysis. Revista Brasileira de Enfermagem, 71(1):135-41. https://doi.org/10.1590/0034-7167-2016-0019.

Vilaço, R. L. B., Gonçalves, D., Silva, V. F. da, \& Vetorazo, J. V. P. (2021). Fatores que levam a alta incidência da síndrome de Burnout nos profissionais da enfermagem. Revista Eletrônica Acervo Enfermagem, 12, e7894. https://doi.org/10.25248/reaenf.e7894.2021

Zhang, W. R., Wang, K., Yin, L., Zhao, W. F., Xue, Q., Peng, M., Min, B. Q., Tian, Q., Leng, H. X., Du, J. L., Chang, H., Yang, Y., Li, W., Shangguan, F. F., Yan, T. Y., Dong, H. Q., Han, Y., Wang, Y. P., Cosci, F., \& Wang, H. X. (2020). Mental Health and Psychosocial Problems of Medical Health Workers during the COVID-19 Epidemic in China. Psychotherapy and psychosomatics, 89(4), 242-250. https://doi.org/10.1159/000507639 